\title{
Immunologie
}

\section{Zellen des Immunsystems: vom Polarkreis ins All}

\author{
LILIANA E. LAYER ${ }^{1}$, OLIVER ULLRICH ${ }^{2}$ \\ ${ }^{1}$ PROMOCELL GMBH, HEIDELBERG \\ ${ }^{2}$ ANATOMISCHES INSTITUT UND ZURICH CENTER FOR INTEGRATIVE HUMAN \\ PHYSIOLOGY (ZIHP), UNIVERSITÄT ZÜRICH, SCHWEIZ; INSTITUT FÜR MASCHINEN- \\ KONSTRUKTION, FAKULTÄT FÜR MASCHINENBAU, UNIVERSITÄT MAGDEBURG
}

\section{Das Immunsystem gehört auf einem Raumflug zu den am stärksten beeinträchtigten Systemen des menschlichen Körpers. Die Ursache dieser Störung liegt wahrscheinlich auf Ebene der Zellen. Experimente an Bord einer TEXUS-Forschungsrakete sollen nun Aufschluss hierüber geben.}

Immune system malfunction is one of the major limiting factors for human health and performance during space flight. Experiments on board of the TEXUS-49 rocket were carried out to shed light on the underlying molecular alterations.

\section{Das „Immunproblem“ im All}

Das Immunsystem gehört zu den auf einem Raumflug mit am stärksten beeinträchtigten Systemen des menschlichen Körpers [1]. Infektionen des Respirationstrakts, des Mundraums, der Augen, der Harnwege und der Haut begleiten die Geschichte des bemannten Raumfluges seit den ersten Apollo-Missionen. Studien zeigten bei Astronauten einen Anstieg der Antikörpertiter gegen Epstein-Barr- und Cytomegalie-Virus sowie eine sprunghafte Reaktivierung des Varizella-Zoster-Virus [2].

Bei der Analyse des Immunstatus fallen auf Ebene der Zellzahlen nur einige und eher wenig dramatische Veränderungen auf, während aber Funktionstests ein dramatisches Bild zeigen: Die proliferative Kapazität von T-Lymphozyten, die zytotoxische Aktivität von NK-Zellen, die Phagozytoseaktivität und die oxidative burst-Reaktion (Bildung reaktiver Sauerstoffspezies) im Monozyten/Makrophagensystem sinken massiv ab. Schwerelosigkeit führt zu einer komplexen Funktionsstörung in Zellen des Immunsystems, die sowohl die spezifische wie auch die innate Immunität und die Immuneffektorfunktionen betrifft. Vor dem Hintergrund einer erhöhten Virulenz und Resistenzentwicklung pathogener Bakterienstämme unter Weltraumbe- dingungen und der Tatsache, dass sich virale und bakterielle Infektionen rapide an Bord ausbreiten können, kann von einer existierenden Gefährdung des Astronauten durch Störungen des Immunsystems im All ausgegangen werden.

Ein Großteil dieses Problems liegt wahrscheinlich in der direkten Wirkung veränderter Schwerkraft auf Zellebene. Viele Studien lieferten bisher Hinweise auf zelluläre und molekulare Funktionsstörungen in Zellen

- Abb. 1: Flugprofil der TEXUS. Der Start wird vom ESRANGE Space Center, ca. 150 Kilometer nördlich des Polarkreises bei Kiruna, Schweden, durchgeführt. Innerhalb der Nutzlast, die befördert wird, befinden sich die Experimente, die mittels Telecommand während des Fluges überwacht und gesteuert werden. Nach Trennung des Antriebs von der Nutzlast wird die zuvor zur Stabilisierung des Fluges aufgebaute Rotation der Rakete gebremst. Ab einer Höhe von 100 Kilometern herrscht an Bord der TEXUS Mikrogravitation. Der Scheitelpunkt des Fluges wird in einer Höhe von 250 bis 300 Kilometern erreicht (Low Earth Orbit, LEO) und danach folgt der freie Fall zurück zur Erde. Die Nutzlast wird anschließend geborgen und per Helikopter zum ESRANGE Space Center zurücktransportiert (Bild: EADS Astrium N. V.). von einem zweistufigen Trägersystem ins All des Immunsystems, allerdings ohne dass bisher die primären molekularen Ursachen identifiziert werden konnten [3]. Zelluläre Schwerkraftwahrnehmung ist wahrscheinlich nicht die Folge der direkten Aktivierung eines Schwerkraft-wahrnehmenden einzelnen Moleküls, sondern Folge der Änderung der Kraftwirkung auf die extrazelluläre Matrix, der Zellform, der Zytoskelettorganisation oder der inneren Vorspannung der mechanischen Elemente der Zelle.

\section{Hämatopoietische Stammzellen und myelomonozytäre Zellen mit TEXUS- 49 im All}

TEXUS steht für „Technologische Experimente unter Schwerelosigkeit“ und bezeichnet ein 1976 ins Leben gerufenes Forschungsraketen-Programm, das auf dem europäischen Raketenstartplatz ESRANGE (European Space und Sounding Rocket Range), nördlich des

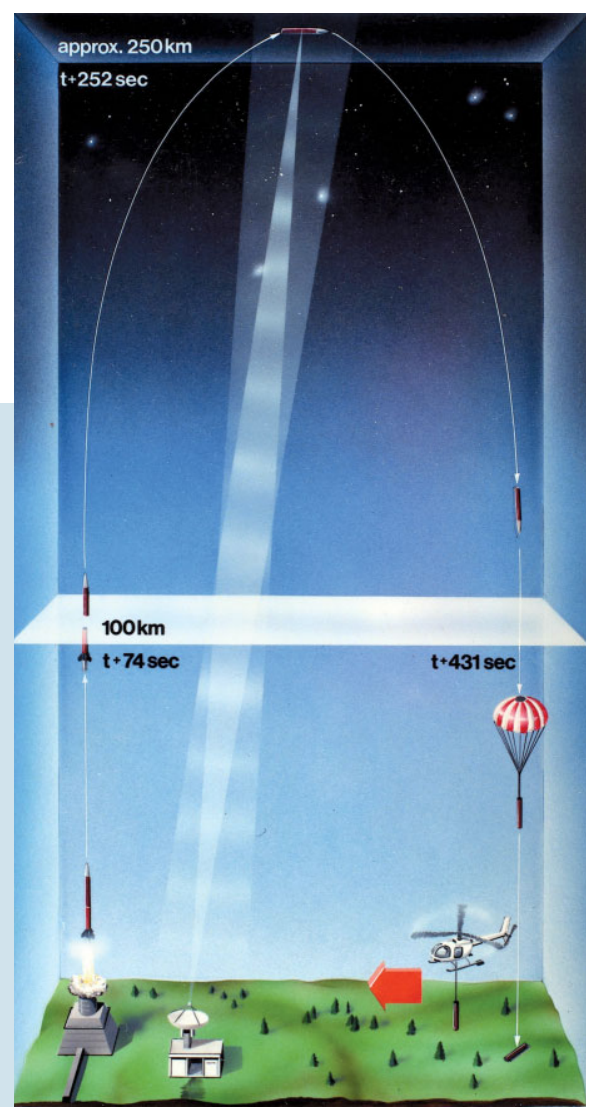




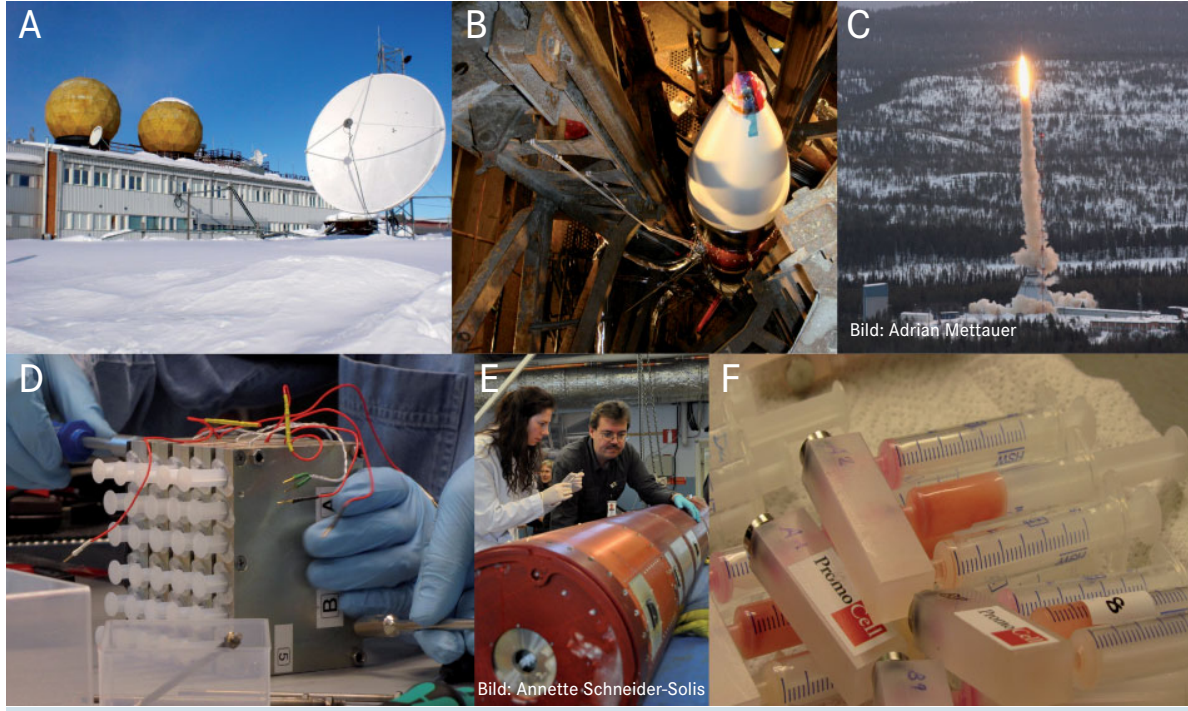

$\Delta$ Abb. 2: Das Experiment Signal Transduction in Cells of the Immune System in Microgravity zur Untersuchung der gesamt-genomischen Expression in Zellen an Bord der Forschungsrakete TEXUS-49. Am ESRANGE Space Center (A), Kiruna, Schweden, wurde TEXUS-49 (B) mit dem Versuch an Bord am 29. März 2011 um 06.01 Uhr gestartet (C). CD 133+-Progenitor- und U937-Zellen wurden in ein temperiertes Experimentmodul (D) überführt, das eine Stunde vor Start in die Rakete eingebaut wurde (E). Nach einem 20-minüten Flug und fünf Minuten Experimentierzeit in Schwerelosigkeit wurde die Nutzlast mittels Helikopter geborgen und die Spritzenmodule mit den Zellen aus der Rakete ausgebaut (F), die RNA vor Ort isoliert und in flüssigem Stickstoff zurücktransportiert.

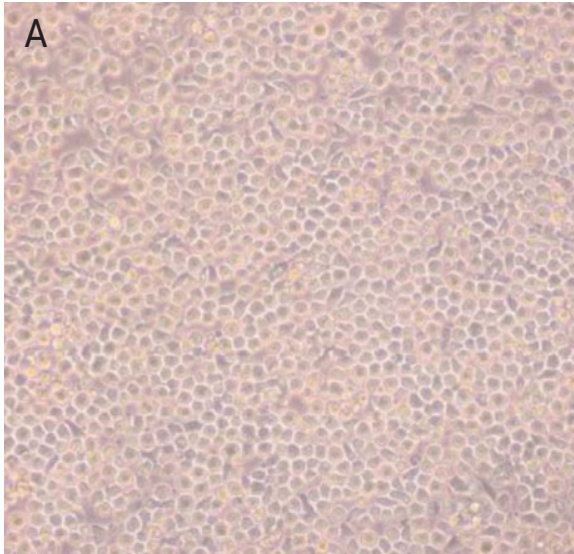

$100 \mu \mathrm{m}$

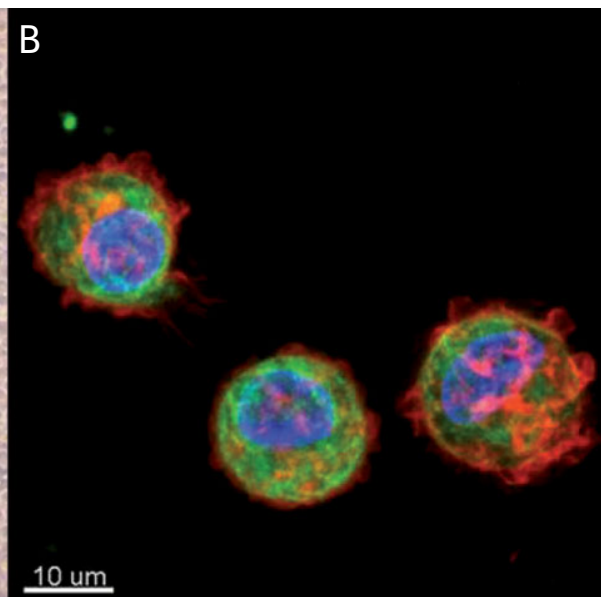

A Abb. 3: Zellen als Modellsystem zur Untersuchung von molekularen Mechanismen unter Schwerelosigkeit. A, Humane CD $133^{+}$-Progenitorzellen, Durchlichtaufnahme einer expandierten Kultur. B, Humane myelomonozytäre U937-Zellen, Fluoreszenzfärbung. Grün: Zytoskelett (Marker Vimentin); rot: F-Aktin (Marker Phallodin); blau: Zellkerne (Marker DAPI).

Polarkreises bei Kiruna, Schweden, in Kooperation des Deutschen Zentrums für Luft- und Raumfahrt (DLR) mit EADS Astrium und der Schwedischen Weltraumagentur (SSC) durchgeführt wird. Es dient unter anderem der Vorbereitung von Experimenten auf der Internationalen Raumstation ISS und ermöglicht biologische, materialwissenschaftliche und physikalische Experimente unter Weltraumbedingungen. Bei den Forschungsraketen des TEXUS-Programms handelt es sich um eine ca. 13 Meter lange, zweistufige Feststoffrake- te (VSB-30), auf der eine ca. fünf Meter lange und bis zu 400 Kilogramm schwere Nutzlast sitzt. Während des ballistischen Fluges wird eine Höhe von bis zu 300 Kilometern mit einer etwa sechsminütigen Schwerelosigkeitsphase erreicht (Abb. 1).

Auf der 16. DLR-Parabelflugkampagne im November 2010 in Bordeaux gelang uns erstmalig eine gesamt-genomische Analyse aller Gene in einer humanen T-Zelllinie, die zur Identifikation einer Vielzahl schwerkraftsensitiver Expressionsänderungen führte. Die
DNA-Mikroarray-Analyse zeigte, dass eine große Anzahl an Genen ein differenzielles Expressionsprofil bei einem Null-Gramm-versus-ein-Gramm-Vergleich aufweist. Das in dieser Kampagne gewonnene „Gravitom“ zeigte nach 20 Sekunden Schwerelosigkeit deutliche Änderungen in der Expression von Genen der Tubuline und des ARP(actin related protein)2/3-Komplexes. Auch fanden sich deutliche Schwerkraft-abhängige Effekte in der Expression von Genen der Zellzyklusregulatoren (z. B. von CDK4, CDK8, CDK9) sowie auf Gene der MAP-Kinase-Kinase-Familie (z. B. MAP2K2, MAPKAPK3).

Ziel des Experiments auf der TEXUS-49Mission ist die gesamt-genomische Expressionsanalyse in menschlichen Immunzellen nach fünf Minuten Schwerelosigkeit, um richtungsführende Hinweise auf zugrunde liegende molekulare Mechanismen zu erhalten. Insbesondere wurde in diesem Projekt auch die Bedeutung einer Gruppe von Ionenkanälen für schwerkraftsensitive Reaktionen getestet.

An Bord der TEXUS-49, im Versuchsmodul TEM 06-16, wurde das Experiment Signal Transduction in Cells of the Immune System in Microgravity (SITI-1) der Universitäten Magdeburg und Zürich durchgeführt (Abb. 2). Der Versuchsaufbau der 16. DLR-Parabelflugkampagne war analog. Hier kamen sowohl normale humane CD133+-Progenitorzellen (PromoCell) zum Einsatz, als auch humane myelomonozytäre Zellen der Linie U937 (Abb. 3). Die Zelltypen dienen als Modellsystem zur Identifikation von zellulären und molekularen Veränderungen in Schwerelosigkeit. Neben der weit verbreiteten Zelllinie U937 wurden Parallelansätze mit primären Zellen durchgeführt. Da Monozyten - die eigentlichen Analoga der U937-Zellen - nicht über einen Zeitraum von mehreren Tagen kultivierbar und damit für die Mission ungeeignet sind, wurden CD133positive, hematopoietische Stammzellen verwendet. Diese Vorläuferzellen von Monozyten und anderen Blutzellen sind im PromoCellMediensystem auf das bis zu 350-fache der ursprünglichen Zellzahl expandierbar. Die Experimentgruppen bestanden aus einer inflight-baseline-Kontrolle (Fixierung im Flug direkt vor Beginn der Schwerelosigkeit), der in-flight-Mikrogravitation-Experimentgruppe, bei der auch in einem Inhibitorexperiment bestimmte Ionenkanäle getestet wurden, sowie einer Bodenkontrolle, die die Wirkung des generellen „Flugstresses“ kontrolliert. Jede Experimentgruppe besteht aus fünf parallelen Einzelexperimenten. 
Für die Versuchsdurchführung wurden alle Zellen mit einer Konzentration von $5 \times 10^{7}$ Zellen pro Milliliter in Hematopoietic Progenitor Cell Expansion Medium DXF bzw. RPMI Medium (PromoCell) aufgenommen und in insgesamt 35 einzelne Experimentsysteme überführt. Diese ermöglichten die vorprogrammierte Zugabe von Medium mit Testsubstanz und die Zugabe von Trizol-LS zur Zelllyse und RNA-Stabilisierung vor der Landung. Die Einzelsysteme wurden in ein exakt auf $37{ }^{\circ} \mathrm{C}$ temperiertes Experimentmodul integriert, das eine Stunde vor Start über einen late access-Einschub in die startbereite Rakete eingebaut wurde.

Der ca. 20-minütige Flug von TEXUS-49 führte in eine Höhe von 268 Kilometern und setzte dabei die im Experimentmodul beherbergten humanen Zellen des Immunsystems einer rund sechsminütigen Mikrogravitation aus. An der Landestelle wurde zuerst das Experimentmodul ausgebaut und mit einem Helikopter zur ESRANGE-Basis transportiert, wo sofort vor Ort die RNA aus allen Proben isoliert wurde, in flüssigen Stickstoff eingefroren und mittels Helikopter und Flugzeug noch am selben Tag in die Labors an den Heimatuniversitäten zurücktransportiert wurde.

Die erste Auswertung ergab, dass pro Probe eine RNA-Ausbeute zwischen 198 und 280 Mikrogramm und elektrophoretisch eine
RNA integrity number (RIN) zwischen 8,6 und 10 erreicht wurde. Somit gelang es - aufgrund der optimalen Versuchsbedingungen und Logistik während der TEXUS-49-Kampagne - RNA von ausgezeichneter Qualität und Ausbeute zu erhalten. Die Expressionsprofile der TEXUS-49-Mission werden derzeit mittels DNA-Mikroarrays analysiert.

Aus den Ergebnissen erhoffen wir uns einen großen Schritt vorwärts im Verstehen der molekularen Vorgänge, die Schwerelosigkeit auf Zellebene auslöst. Wir hoffen auch auf Hinweise, ob menschliche Zellen in der Lage sind, unter den Bedingungen veränderter Schwerkraft zu leben, zu funktionieren und sich anzupassen. Es kann durchaus sein, dass die optimal an die Erde angepasste zelluläre und molekulare Architektur des Lebens ein Problem für das Leben außerhalb der Erde und in der Schwerelosigkeit darstellt.

\section{Literatur}

[1] Guéguinou N, Huin-Schohn C, Bascove M et al. (2009) Could spaceflight-associated immune system weakening preclude the expansion of human presence beyond Earth's orbit? J Leukoc Biol 86:1027-1038

[2] Mehta SK, Cohrs RJ, Forghani B et al. (2004). Stress-induced subclinical reactivation of varicella zoster virus in astronauts. J Med Virol 72:174-179

[3] Studer M, Bradacs G, Hilliger A et al. (2011) Parabolic maneuvers of the Swiss Air Force fighter jet F5-E as a new research platform for cell culture experiments in microgravity. Acta Astronaut 68:1729-1741

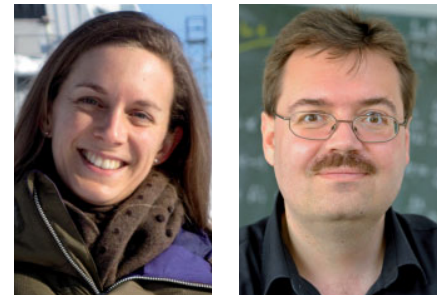

Liliana E. Layer und Oliver Ullrich

Korrespondenzadressen:

Prof. Hon.-Prof. Dr. Dr. Oliver Ullrich

Anatomisches Institut, Universität Zürich

Winterthurerstraße 190

CH-8057 Zürich

Tel.: +41-(0)44-63-55310

Fax: +41-(0)44-63-55498

oliver.ullrich@anatom.uzh.ch

Institut für Maschinenkonstruktion

Fakultät für Maschinenbau

Otto-von-Guericke-Universität Magdeburg

Universitätsplatz 2

D-39106 Magdeburg

Tel.: 0391-67-18522

Fax: 0391-67-12595

oliver.ullrich@ovgu.de

Liliana E. Layer

PromoCell GmbH

Sickingenstraße 63/65

D-69126 Heidelberg

Tel.: 0800-776-6623

Fax: 0800-100-8306

liliana.layer@promocell.com 